\title{
ARTICLE OPEN \\ An effective method to screen sodium-based layered materials for sodium ion batteries
}

\author{
Xu Zhang ${ }^{1}$, Zihe Zhang ${ }^{1}$, Sai Yao ${ }^{1}$, An Chen $^{1}$, Xudong Zhao ${ }^{1}$ and Zhen Zhou (D)
}

Due to the high cost and insufficient resource of lithium, sodium-ion batteries are widely investigated for large-scale applications. Typically, insertion-type materials possess better cyclic stability than alloy-type and conversion-type ones. Therefore, in this work, we proposed a facile and effective method to screen sodium-based layered materials based on Materials Project database as potential candidate insertion-type materials for sodium ion batteries. The obtained Na-based layered materials contains 38 kinds of space group, which reveals that the credibility of our screening approach would not be affected by the space group. Then, some important indexes of the representative materials, including the average voltage, volume change and sodium ion mobility, were further studied by means of density functional theory computations. Some materials with extremely low volume changes and Na diffusion barriers are promising candidates for sodium ion batteries. We believe that our classification algorithm could also be used to search for other alkali and multivalent ion-based layered materials, to accelerate the development of battery materials.

npj Computational Materials (2018)4:13; doi:10.1038/s41524-018-0070-2

\section{INTRODUCTION}

Large-scale energy storage systems for the grid are extremely important for the storage and utilization of the renewable resources, such as solar and wind power., ${ }^{1,2}$ Electrochemical energy storage could satisfy the demand of different grid functions. ${ }^{3}$ Currently, lithium-ion batteries (LIBs) have become the most widely used devices for energy storage. ${ }^{4,5}$ However, the high cost and insufficient resource of lithium inhibit the applications of LIBs in the large-scale electrochemical energy storage. ${ }^{6}$ Sodium-ion batteries (SIBs) are extensively investigated as the most promising alternatives to LIBs due to the natural abundance and appropriate electrode potential. ${ }^{1,7,8}$ However, the ionic radius of $\mathrm{Na}^{+}$is $55 \%$ larger than that of $\mathrm{Li}^{+}$, requiring the host framework with larger space for the storage and transport of $\mathrm{Na}^{+}$. Therefore, simply replacing the electrode materials for SIBs with those for LIBs is impracticable, which makes seeking for eligible $\mathrm{Na}^{+}$host materials extremely important.

Some typical insertion-type electrode materials, such as $\mathrm{Na}_{0.44} \mathrm{MnO}_{2}, \quad \mathrm{Na}_{\mathrm{x}} \mathrm{CoO}_{2},{ }^{9} \quad \mathrm{Na}_{0.66} \mathrm{Co}_{0.5} \mathrm{Mn}_{0.5} \mathrm{O}_{2},{ }^{10} \quad \mathrm{Li}_{4} \mathrm{Ti}_{5} \mathrm{O}_{12},{ }^{11}$ and $\mathrm{Na}_{2} \mathrm{Ti}_{3} \mathrm{O}_{7}$, ${ }^{12}$ were widely investigated owing to their superior cyclic stability to the alloy-type and conversion-type ones. ${ }^{13}$ Among them, Na-containing layered materials have attracted extensive attention since the layered structure ensures the reversible intercalation/de-intercalation of $\mathrm{Na}^{+}$without serious lattice changes, leading to good cyclic stability and excellent ionic conductivity. By means of density functional theory (DFT) computations, many previous reports proposed various electrode materials for LIBs, SIBs, and multivalent ion batteries, which greatly promoted their development. ${ }^{14-17}$ Recently, with the fast development of high-performance computations, high-throughput computational materials design has attracted much attention and improved the efficiency of materials design, further accelerating the investigations on materials for various fields, especially LIBs. ${ }^{18-22}$

Therefore, in this work, more than 60,000 inorganic compounds in Materials Project (MP) Database $e^{23,24}$ were investigated to search for sodium-based layered materials which can potentially be applied as insertion-type electrode materials for SIBs. Then by means of DFT computations, some important indexes of the representative materials, including the average voltage, volume change and sodium ion mobility, were examined. Besides Nabased layered materials, we believe that other alkali and multivalent ion-based layered materials can also be searched by using this method, which might favor the development of electrode materials for batteries.

\section{RESULTS AND DISCUSSION}

Our screening approach for Na-based layered materials is shown in Fig. 1. After the materials screening, over 150 kinds of Na-based layered materials were obtained. The obtained Na-based layered materials contain 38 kinds of space group, as shown in Fig. 2, which belongs to six kinds of crystal systems except the cubic. The results indicate that the accuracy of our screening approach would not be affected by the space group of candidate materials.

The structures of some representative $\mathrm{Na}$-based layered materials are shown in Fig. 3. Na atoms in these materials are mainly distributed between two layers, indicating that $\mathrm{Na}$ atoms could intercalate/de-intercalate with low energy barriers and therefore, these materials can be potentially used as insertion-type electrode materials for SIBs. The results further suggest that our screening approach is highly efficient and credible. This classification algorithm could also be employed to search for other alkali and multivalent ion-based layered materials as potential insertiontype electrode materials.

\footnotetext{
${ }^{1}$ School of Materials Science and Engineering, National Institute for Advanced Materials, Computational Centre for Molecular Science, Institute of New Energy Material Chemistry, Collaborative Innovation Center of Chemical Science and Engineering (Tianjin), Nankai University, Tianjin 300350, China

Correspondence: Zhen Zhou (zhouzhen@nankai.edu.cn)
}

Received: 2 December 2017 Revised: 8 February 2018 Accepted: 21 February 2018

Published online: 20 March 2018 


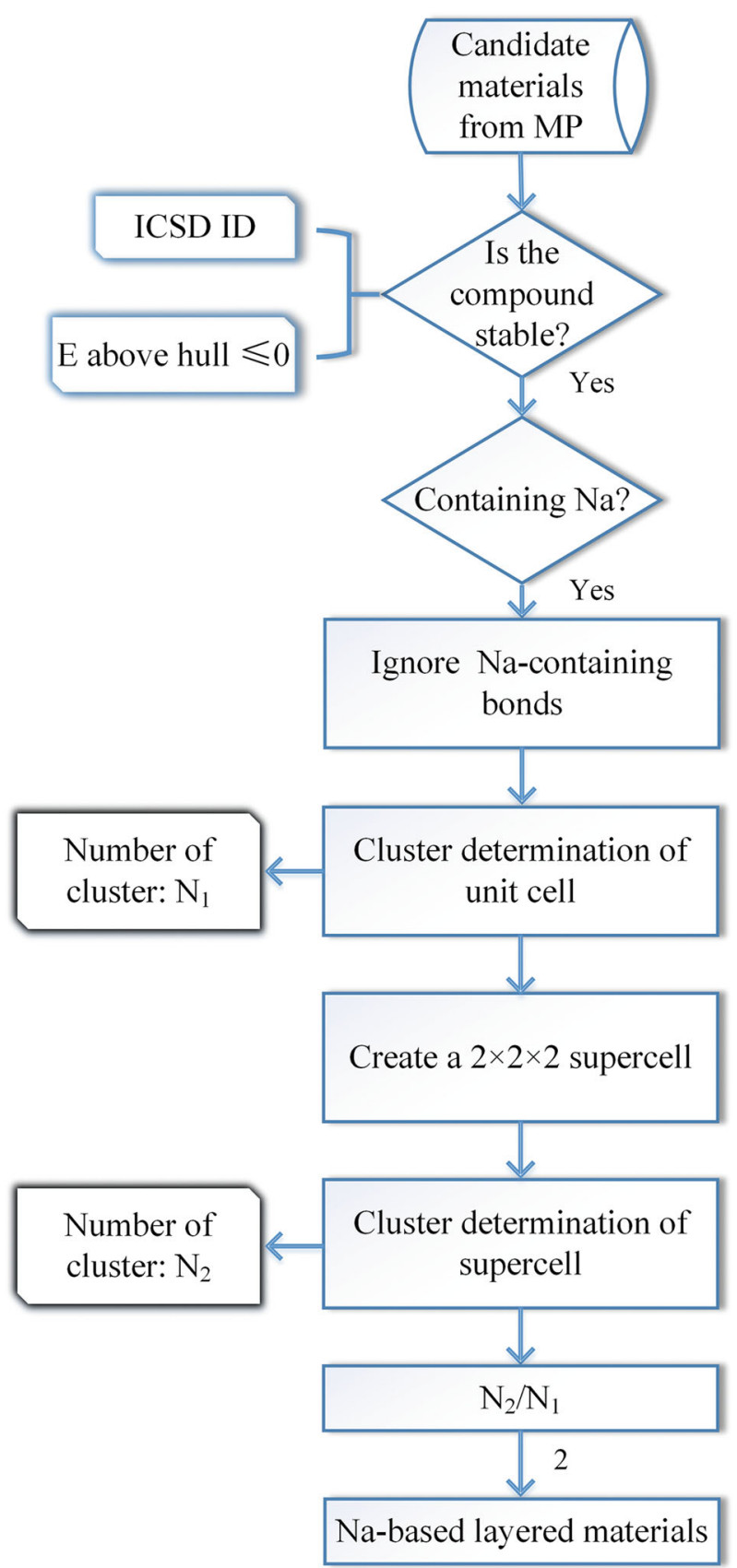

Fig. 1 Flow diagram of the screening approach for Na-based layered materials

To further verify the effectiveness of this method to explore electrode materials for SIBs, some important indexes of representative materials, including the average voltage, volume change and sodium ion mobility were further investigated. The geometries were firstly optimized and the lattice constants are shown in Supplementary Table 1, which are consistent with the experimental data. Some experimentally widely investigated layered materials for SIBs, such as Na-based layered transition metal oxides (TMOs), $\mathrm{NaMO}_{2}$ ( $\mathrm{M}=\mathrm{Co}, \mathrm{Fe}, \mathrm{V}, \mathrm{Ni}$, etc), ${ }^{13,25,26}$ were successfully achieved through screening. Na-based layered TM sulfides, $\mathrm{NaMS}_{2}(\mathrm{M}=\mathrm{Ti}, \mathrm{Sc}, \mathrm{Nb}$, etc), which have similar structures to TMOs, were also obtained, as well as layered TM fluorides, which could possess higher electrode potentials.

Then, the average intercalation voltages were computed based on the reaction energy of $\mathrm{Na}_{n}+\mathrm{X} \rightarrow \mathrm{Na}_{n} \mathrm{X}$, where $\mathrm{n}$ is the number of intercalating $\mathrm{Na}$, and $\mathrm{X}$ is the rest of the compounds. The average intercalation voltage and volume change of the representative Na-based layered compounds are shown in Fig. 4 and Supplementary Table 2. The volume change is a critical parameter for electrode materials during $\mathrm{Na}$ intercalation/deintercalation. Large volume changes could lead to the tremendous damage to electrodes, causing the loss of electrical contact and rapid capacity fading. Compared with alloy- and conversion-type electrode materials, the superior cyclic stability of insertion-type materials lies in much lower volume change during Na insertion/ extraction. As shown in Fig. 4 and Supplementary Table 2, the volume changes of most materials are smaller than $25 \%$, and for $\mathrm{NaTiF}_{4}$ and $\mathrm{Na}_{2} \mathrm{Cu}\left(\mathrm{CO}_{3}\right)_{2}$, minor volume changes of $13 \%$ imply good cyclic stability.

However, for some materials as shown in Supplementary Fig. 1, the volume change is higher than $50 \%$. After $\mathrm{Na}^{+}$de-intercalation, the interlayer spacing decreases dramatically, causing large volume change and collapse of the layered structure. To settle the issue, some $\mathrm{Na}$ atoms should be kept in the materials to support the layered structure during the de-intercalation of $\mathrm{Na}$. Besides, introducing heteroatom as pillars is another effective method to improve the structural stability. ${ }^{27}$ The average intercalation voltages of many representative materials range from 2.5 to $4.5 \mathrm{~V}$, which indicates that they have the potential to be used as cathode materials for SIBs.

The corresponding gravimetric capacity is also listed in Supplementary Table 2 . It is important to note that the gravimetric capacity was computed based on the number of $\mathrm{Na}$ that the materials naturally possess. In experiments, some materials could accommodate extra $\mathrm{Na}$ atoms. For example, recently, $\mathrm{Na}_{2} \mathrm{Mn}_{3} \mathrm{O}_{7}$ has been investigated as an electrode material for SIBs, and the results indicate that it could reversibly insert and extract two extra $\mathrm{Na}$ atoms. ${ }^{28} \mathrm{Na}_{2} \mathrm{Ti}_{3} \mathrm{O}_{7}$, a widely investigated $\mathrm{SIB}$ electrode material, could also accommodate more $\mathrm{Na}$ atoms. ${ }^{12}$

Besides the materials that have been investigated for SIBS before, many materials are proposed as potential insertion-type electrode materials. Metal fluorides possess high average voltage and low volume change, especially $\mathrm{NaVF}_{4}$ with an average voltage of $4.30 \mathrm{~V}$, which would be promising candidates for cathode materials of SIBs. $\mathrm{Na}_{2} \mathrm{Cu}\left(\mathrm{CO}_{3}\right)_{2}$, which has a low volume change of $13 \%$, and a high average voltage of $4.39 \mathrm{~V}$, can also be potentially applied as a cathode material. Some other materials, such as $\mathrm{Na}_{2} \mathrm{Zr}$ $\left(\mathrm{CuS}_{2}\right)_{2}, \mathrm{Na}(\mathrm{CuO})_{2}$, and $\mathrm{Na}_{3} \mathrm{Co}_{2} \mathrm{SbO}_{6}$, were also found with appropriate voltage and low volume change.

The rate performance of SIB electrode materials heavily depends on $\mathrm{Na}$ ion mobility; therefore, it is important to estimate the diffusion of $\mathrm{Na}$ in the interlayer. The climbing-image nudged elastic band (Cl-NEB) method, which has been successfully applied in the study of ion diffusion, ${ }^{17,29-31}$ was adopted to simulate the migration of $\mathrm{Na}$. The energy profiles and barriers are shown in Fig. 5 and Table 1. Due to their special layered structure, the diffusion barriers of $\mathrm{Na}$ in the interlayer are quite low. The computed diffusion barrier of $\mathrm{Na}$ in $\mathrm{NaCoO}_{2}$ is only $0.31 \mathrm{eV}$, which is consistent with those in previous reports. ${ }^{32,33}$ For Na-based layered TM sulfides, the diffusion barriers are even lower $\left(0.22 \mathrm{eV}\right.$ for $\mathrm{NaScS}_{2}$ and $0.19 \mathrm{eV}$ for $\left.\mathrm{NaTiS}_{2}\right)$, which could be attributed to the lower electronegativity of $S$ atoms. However, for some materials, such as NaAlSi, the diffusion barriers are higher than $0.6 \mathrm{eV}$, which can be ascribed to the large volume change.

Then, the electronic conductivity can be evaluated based on the band gaps obtained from MP database in Table 1 and the density of states in Supplementary Fig. 2. Among them, $\mathrm{Na}_{2} \mathrm{Ti}_{3} \mathrm{O}_{7}$ possesses the largest band gap of $2.99 \mathrm{eV}$, which would cause rather low electronic conductivity. In experiments, some promising solutions could be applied to overcome this challenge, such as chemical modification and morphology control. ${ }^{12,34,35}$ Besides, the band gaps of other materials are lower than that of $\mathrm{Na}_{2} \mathrm{Ti}_{3} \mathrm{O}_{7}$, indicating better electronic conductivity. The partial density of 


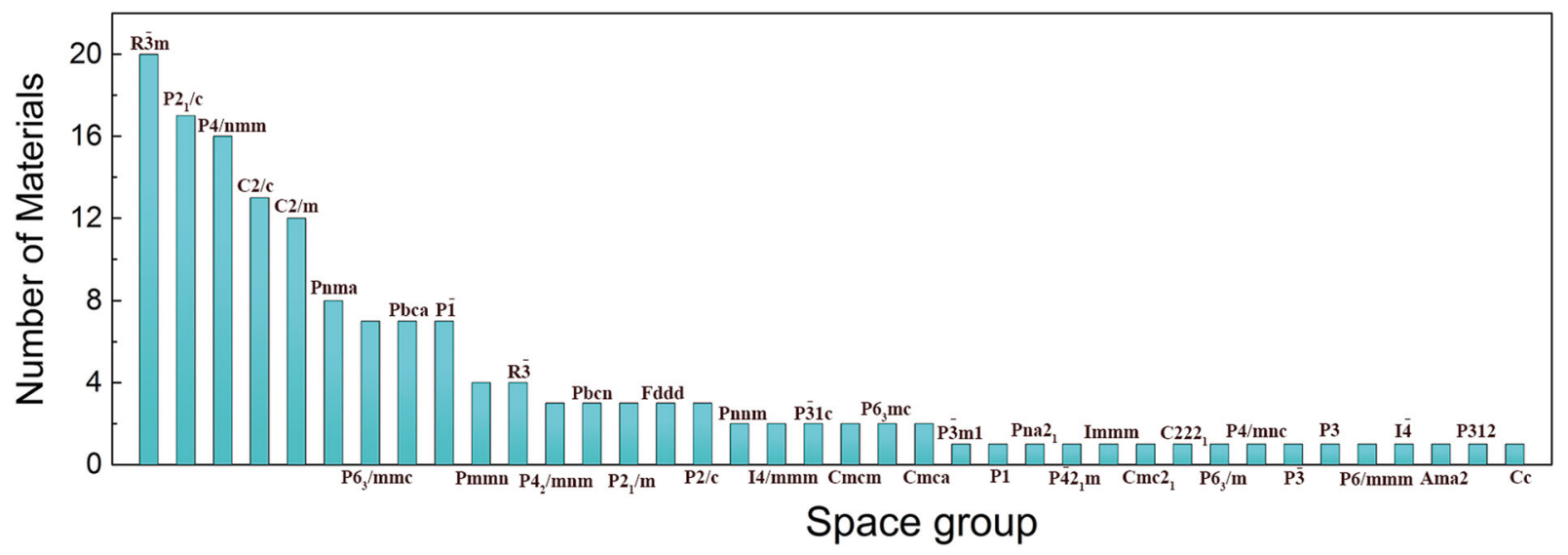

Fig. 2 Distribution of space groups for the obtained Na-based layered materials

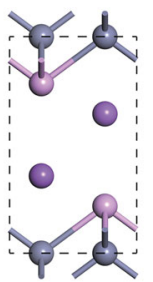

$\mathrm{NaPZn}$

$\mathrm{mp}-4824$

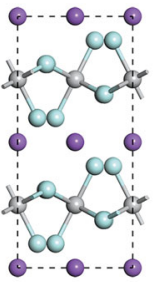

$\mathrm{NaTiF}_{4}$ mp-27264

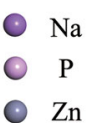

- $\mathrm{Na}$

- $\mathrm{Ti}$

O F mp-505090
$\mathrm{Na}(\mathrm{CuO})_{2}$

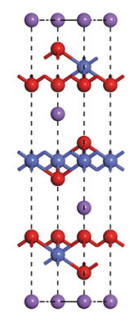

$\mathrm{NaCoO}_{2}$ mp-18921

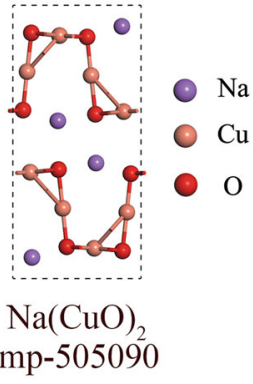

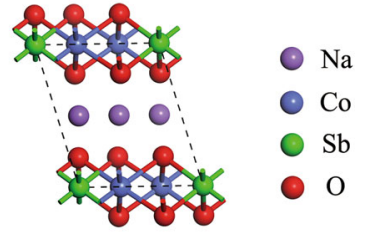

$\mathrm{Na}_{3} \mathrm{Co}_{2} \mathrm{SbO}_{6}$

mp-19087

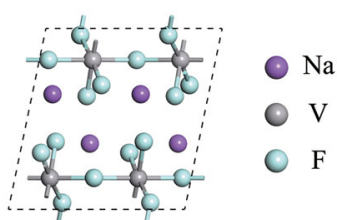

$\mathrm{NaVF}_{4}$ $\mathrm{mp}-559187$

Fig. 3 Structures of some representative Na-based layered materials. The purple balls represent $\mathrm{Na}$

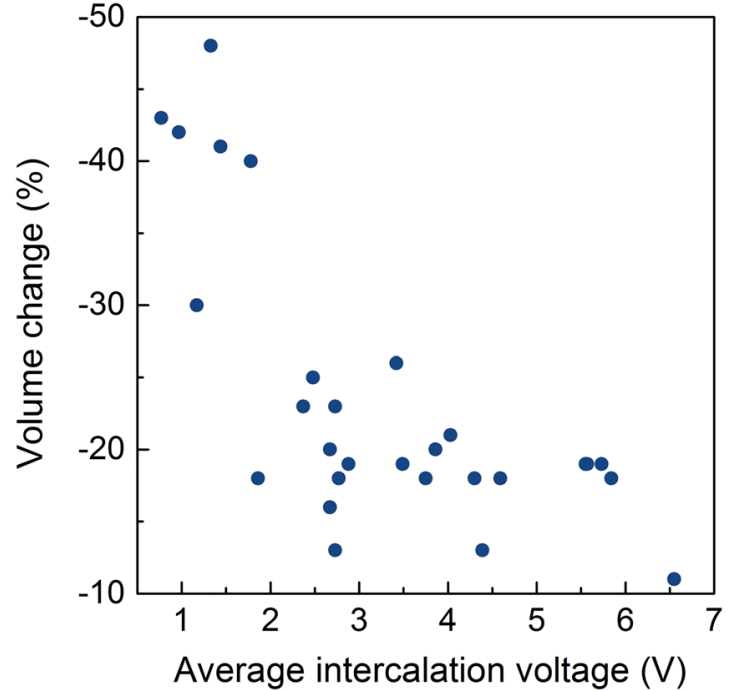

Fig. 4 The volume change after the de-intercalation of $\mathrm{Na}$ vs. average intercalation voltage states (PDOS) reveals that $\mathrm{Na}$ contributes little to the states near the Fermi level. For some materials, such as $\mathrm{NaTiS}_{2}$, the high peaks around the Fermi level imply high-concentration carriers and thus good electronic conductivity.

Based on the computed average voltage, volume change, gravimetric capacity, diffusion barrier and band gap, the electrochemical performance of representative Na-based layered materials for SIBs could be predicted. Compared with alloy-type and conversion-type materials, Na-based layered materials possess lower volume change after the de-intercalation of $\mathrm{Na}$. The volume change of most materials is lower than $25 \%$. Besides, the diffusion barrier of $\mathrm{Na}$ in the interlayer is extremely low, indicating excellent rate performance. In this work, we proposed some insertion-type electrode materials for $\mathrm{SIBs}$, such as $\mathrm{Na}(\mathrm{CuO})_{2}, \mathrm{NaTiF}_{4}, \mathrm{Na}_{2} \mathrm{Zr}$ $\left(\mathrm{CuS}_{2}\right)_{2}, \mathrm{Na}_{3} \mathrm{CO}_{2} \mathrm{SbO}_{6}$, and $\mathrm{Na}_{2} \mathrm{Cu}\left(\mathrm{CO}_{3}\right)_{2}$, which possess appropriate voltage, high capacity, low volume change, Na diffusion barrier and band gap, and can be potentially used as cathode materials for SIBs. Detailed computations about the voltage and volume change for $\mathrm{Na}_{3} \mathrm{CO}_{2} \mathrm{SbO}_{6}$ and $\mathrm{Na}_{2} \mathrm{Cu}\left(\mathrm{CO}_{3}\right)_{2}$ are shown in Supplementary Fig. 3 and Supplementary Table 3. We would like to invite experimental colleagues to explore the practical performances of these materials in SIBs. 

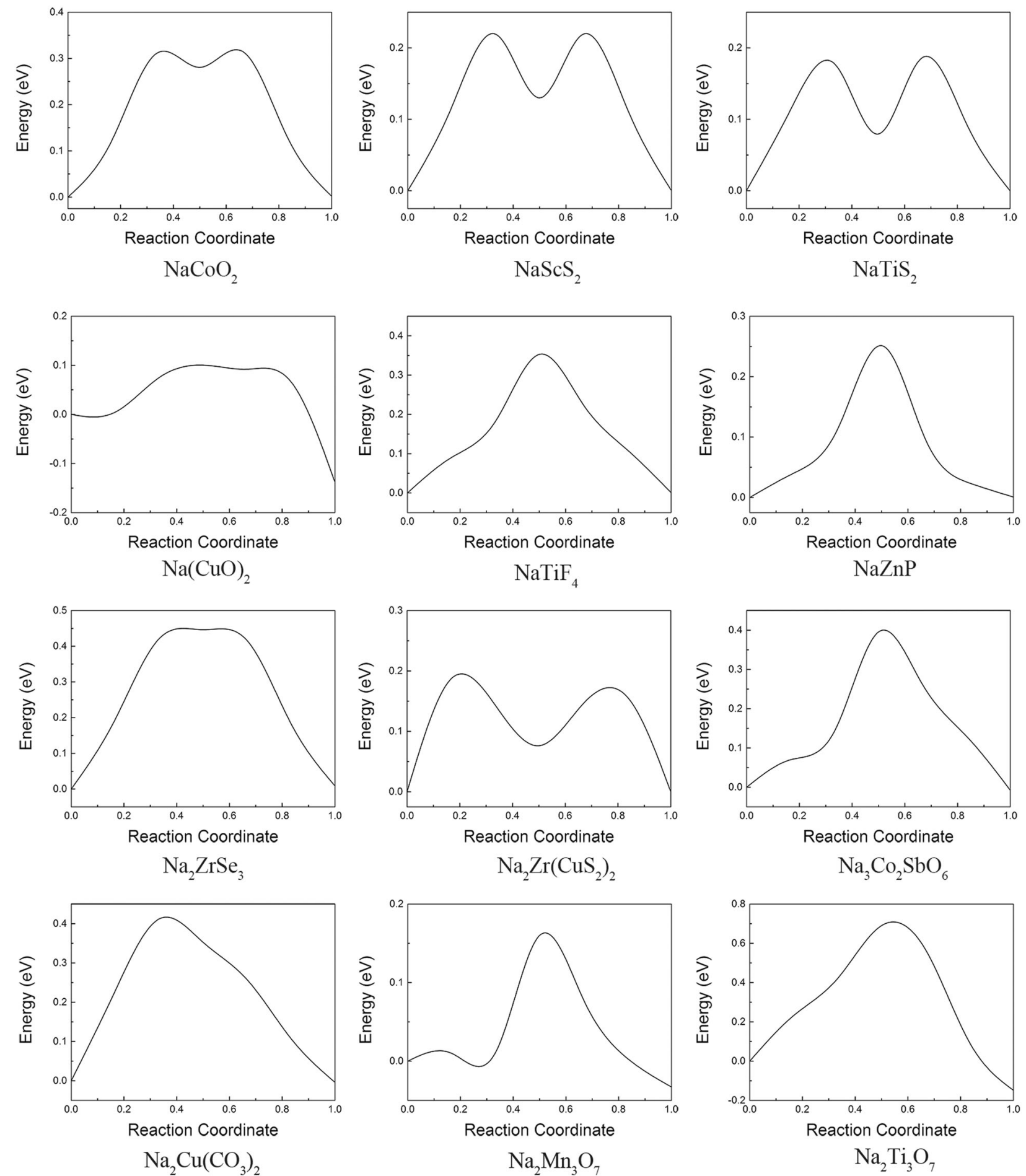

Fig. 5 Calculated minimum energy profiles of Na migration at the interlayer in some representative Na-based layered materials

In conclusion, we proposed a facile and effective method to search for sodium-based layered materials from MP database. The obtained layered materials contain 38 kinds of space group, indicating that our screening approach would not be affected by the space group. Then the average voltage, volume change and $\mathrm{Na}$ ion mobility were further investigated by means of DFT. The results confirm that the volume change and $\mathrm{Na}$ ion diffusion barrier are extremely low, making them promising candidate electrode materials for SIBs. We believe that our method could also be employed to search for other alkali and multivalent ionbased layered compounds as potential insertion-type electrode materials, and further accelerate the development of nextgeneration batteries.

\section{METHODS}

Vienna ab initio simulation package (VASP) was used to perform DFT computations. ${ }^{36}$ The ion-electron interaction was described by using the 
Table 1. The energy barrier $(\mathrm{eV})$ of $\mathrm{Na}$ ion migration in the interlayer and the band gap (eV) for some representative Na-based layered materials

\begin{tabular}{lll}
\hline Formula & Energy barrier & Band gap \\
\hline $\mathrm{NaCoO}_{2}$ & 0.31 & 2.11 \\
$\mathrm{NaScS}_{2}$ & 0.22 & 1.81 \\
$\mathrm{NaTiS}_{2}$ & 0.19 & 0 \\
$\mathrm{Na}(\mathrm{CuO})_{2}$ & 0.10 & 0 \\
$\mathrm{NaTiF}$ & 0.35 & 0.20 \\
$\mathrm{NaZnP}$ & 0.25 & 0.91 \\
$\mathrm{NaAlSi}_{\mathrm{Na}}$ & 0.63 & 0 \\
$\mathrm{Na}_{2} \mathrm{ZrSe}$ & 0.45 & 1.32 \\
$\mathrm{Na}_{2} \mathrm{Zr}\left(\mathrm{CuS}_{2}\right)_{2}$ & 0.19 & 0.01 \\
$\mathrm{Na}_{3} \mathrm{Co}_{2} \mathrm{SbO}_{6}$ & 0.40 & 1.91 \\
$\mathrm{Na}_{2} \mathrm{Cu}_{\left(\mathrm{CO}_{3}\right)_{2}}$ & 0.41 & 0.49 \\
$\mathrm{Na}_{2} \mathrm{Mn}_{3} \mathrm{O}_{7}$ & 0.16 & 1.41 \\
$\mathrm{Na}_{2} \mathrm{Ti}_{3} \mathrm{O}_{7}$ & 0.69 & 2.99 \\
\hline
\end{tabular}

projector augmented wave (PAW) method. ${ }^{37}$ A $520 \mathrm{eV}$ cutoff was applied for the plane-wave basis set. The functional of Perdew, Burke, and Ernzerhof (PBE) was employed to describe the exchange-correlation energy. ${ }^{38}$ In order to accurately deal with the van der Waals force for weak interactions, the DFT-D3 method with Becke-Jonson damping was used. $^{39,40}$ K-point separation of $0.03 \AA^{-1}$ was employed. Considering the strong electron correlation effects, the U-J parameters were applied as the same as those adoped by Materials Project. ${ }^{23}$ Also, magnetic ions were intialized ferromagnetically. The energy barriers of $\mathrm{Na}$ ion migration were computed by the CI-NEB method. ${ }^{41}$ The average voltage was caculated as:

$\mathrm{V}_{\mathrm{ave}}=\left(E_{\mathrm{de}}+n E_{\mathrm{Na}}-E_{\mathrm{int}}\right) /(\mathrm{ne})$

where $E_{\mathrm{de}}, E_{\mathrm{int}}$ and $E_{\mathrm{Na}}$ represent the energy of the Na de-intercalation and intercalation structure, and the energy of $\mathrm{Na}$ atom in the metal crystal, respectively; $n$ is the number of intercalated $\mathrm{Na}$. The volume change was computed according to

$v_{\text {change }}=\left(v_{\text {de }}-v_{\text {in }}\right) / v_{\text {in }}$

where $v_{\text {in }}$ and $v_{\text {de }}$ represent the volume before and after the deintercalation of $\mathrm{Na}$.

The inorganic materials from MP database are the candidates for screening. The stability of the candidate compound was examined by using two criteria, (i) the compound must have an Inorganic Crystal Structure Database (ICSD) ${ }^{42}$ ID, indicating the experimental feasibility; (ii) the energy above convex hull must be zero, which ensures that it is the most energetically stable phase compared with its competing phases. Besides, only the compounds containing $\mathrm{Na}$ were chosen to ensure their capability for $\mathrm{Na}$ accommodation.

We proposed a simple method to search for $\mathrm{Na}$-based layered materials. The covalent bonds in the unit cell of the candidate materials were identified based on:

$$
T_{1} \times\left(r_{\mathrm{i}}^{\mathrm{cov}}+r_{\mathrm{j}}^{\mathrm{cov}}\right)<d_{\mathrm{ij}}<T_{2} \times\left(r_{\mathrm{i}}^{\mathrm{cov}}+r_{\mathrm{j}}^{\mathrm{cov}}\right)
$$

in which $d_{\mathrm{ij}}$ is the distance between the atom $\mathrm{i}$ and $\mathrm{j} ; r_{\mathrm{i}}{ }^{\mathrm{cov}}$ and $r_{\mathrm{j}}{ }^{\mathrm{cov}}$ are the experiential covalent radius of atom $\mathrm{i}$ and $\mathrm{j}$, respectively; and $T_{1}$ and $T_{2}$ represent the lower and upper bond-length tolerance, respectively. In this work, $T_{1}$ and $T_{2}$ were set as 0.6 and 1.15 , respectively, which were also applied as default values in Materials Studio. The $\mathrm{Na}$-containing bonds in the candidate materials were ignored in order to make sure a precise evaluation of the structure of other atoms. Then, the atoms connected by covalent bonds were regarded as a cluster, and the number of clusters in a unit cell was defined as $N_{1}$. Similarly, the number of clusters in a $2 \times 2 \times$ 2 supercell was denoted by $N_{2}$. If $N_{2} / N_{1}=2$, the compounds can be judged to be Na-based layered materials.

\section{Data availability}

The authors declare that the data supporting the findings of this study are available within the paper, and the comparison of the lattice parameters, average intercalation voltage, volume change, gravimetric capacity, atomic structure and density of states for some representative $\mathrm{Na}$-based layered materials and the average voltage profile and volume change of $\mathrm{Na}_{2} \mathrm{Cu}$ $\left(\mathrm{CO}_{3}\right)_{2}$ and $\mathrm{Na}_{3} \mathrm{CO}_{2} \mathrm{SbO}_{6}$ are available in its supplementary information files.

\section{ACKNOWLEDGEMENTS}

This work was supported by NSFC (21773126) in China. The computations were performed on Magic Cube at Shanghai Supercomputer Center.

\section{AUTHOR CONTRIBUTIONS}

X.Z. performed the computations, analyzed the results and wrote the manuscript. Z.H. Z., S.Y., and A.C. advised on computations. X.Z., Z.H.Z., and X.D.Z. proposed and edited the screening algorithm. Z.Z. analyzed the results and supervised the project. All authors reviewed and approved the manuscript.

\section{ADDITIONAL INFORMATION}

Supplementary information accompanies the paper on the npj Computational Materials website (https://doi.org/10.1038/s41524-018-0070-2).

Competing interests: The authors declare no competing financial interests.

Publisher's note: Springer Nature remains neutral with regard to jurisdictional claims in published maps and institutional affiliations.

\section{REFERENCES}

1. Yabuuchi, N., Kubota, K., Dahbi, M. \& Komaba, S. Research development on sodium-ion batteries. Chem. Rev. 114, 11636-11682 (2014).

2. Yang, J., Zhou, X., Wu, D., Zhao, X. \& Zhou, Z. S-doped N-rich carbon nanosheets with expanded interlayer distance as anode materials for sodium-ion batteries. Adv. Mater. 29, 1604108 (2017).

3. Dunn, B., Kamath, H. \& Tarascon, J.-M. Electrical energy storage for the grid: a battery of choices. Science 334, 928-935 (2011).

4. Tarascon, J.-M. \& Armand, M. Issues and challenges facing rechargeable lithium batteries. Nature 414, 359-367 (2001).

5. Zhong, Y. et al. Orderly packed anodes for high-power lithium-ion batteries with super-long cycle life: rational design of $\mathrm{MnCO}_{3} /$ large-area graphene composites. Adv. Mater. 27, 806-812 (2015).

6. Tarascon, J. M. Is lithium the new gold? Nat. Chem. 2, 510 (2010)

7. Zhu, Y.-E. et al. Fast sodium storage in $\mathrm{TiO}_{2} @ C N T @ C$ nanorods for highperformance Na-ion capacitors. Adv. Energy Mater. 1701222 (2017).

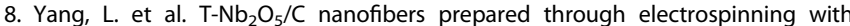
prolonged cycle durability for high-rate sodium-ion batteries induced by pseudocapacitance. Small 1702588 (2017).

9. Palomares, V. et al. Na-ion batteries, recent advances and present challenges to become low cost energy storage systems. Energy Environ. Sci. 5, 5884-5901 (2012).

10. Zhu, Y.-E. et al. A P2- $\mathrm{Na}_{0.67} \mathrm{Co}_{0.5} \mathrm{Mn}_{0.5} \mathrm{O}_{2}$ cathode material with excellent rate capability and cycling stability for sodium ion batteries. J. Mater. Chem. A 4 11103-11109 (2016).

11. Zhou, Q. et al. Synthesis of lithium titanate nanorods as anode materials for lithium and sodium ion batteries with superior electrochemical performance. $J$. Power Sources 283, 243-250 (2015).

12. Pan, $\mathrm{H}$. et al. Sodium storage and transport properties in layered $\mathrm{Na}_{2} \mathrm{Ti}_{3} \mathrm{O}_{7}$ for room-temperature sodium-ion batteries. Adv. Energy Mater. 3, 1186-1194 (2013).

13. Li, F. \& Zhou, Z. Micro/nanostructured materials for sodium ion batteries and capacitors. Small 14, 1702961 (2018).

14. Urban, A., Seo, D.-H. \& Ceder, G. Computational understanding of Li-ion batteries. npj Comput. Mater. 2, 16002 (2016).

15. Islam, M. S. \& Fisher, C. A. Lithium and sodium battery cathode materials: computational insights into voltage, diffusion and nanostructural properties. Chem. Soc. Rev. 43, 185-204 (2014).

16. Bhatt, M. D. \& O'Dwyer, C. Recent progress in theoretical and computational investigations of Li-ion battery materials and electrolytes. Phys. Chem. Chem. Phys. 17, 4799-4844 (2015).

17. Tang, Q., Zhou, Z. \& Shen, P. Are MXenes promising anode materials for Li ion batteries? computational studies on electronic properties and Li storage capability of $\mathrm{Ti}_{3} \mathrm{C}_{2}$ and $\mathrm{Ti}_{3} \mathrm{C}_{2} \mathrm{X}_{2}(\mathrm{X}=\mathrm{F}, \mathrm{OH})$ monolayer. J. Am. Chem. Soc. 134, 16909-16916 (2012). 
18. Hautier, G. et al. Phosphates as lithium-ion battery cathodes: an evaluation based on high-throughput ab initio calculations. Chem. Mater. 23, 3495-3508 (2011).

19. Kirklin, S., Meredig, B. \& Wolverton, C. High-throughput computational screening of new Li-ion battery anode materials. Adv. Energy Mater. 3, 252-262 (2013).

20. Jain, A., Hautier, G., Ong, S. P., Dacek, S. \& Ceder, G. Relating voltage and thermal safety in Li-ion battery cathodes: A high-throughput computational study. Phys. Chem. Chem. Phys. 17, 5942-5953 (2015).

21. Aykol, M. et al. High-throughput computational design of cathode coatings for Liion batteries. Nat. Commun. 7, 13779 (2016).

22. Sendek, A. D. et al. Holistic computational structure screening of more than 12000 candidates for solid lithium-ion conductor materials. Energy Environ. Sci. 10, 306-320 (2017).

23. Jain, A. et al. Commentary: the materials project: a materials genome approach to accelerating materials innovation. APL Mat. 1, 011002 (2013).

24. Ong, S. P. et al. The Materials Application ProgrammingInterface (API): A simple, flexible and efficient API for materials data based on REpresentational State Transfer (REST) principles. Comp. Mater. Sci. 97, 209-215 (2015).

25. Fang, C. et al. Routes to high energy cathodes of sodium-ion batteries. Adv. Energy Mater. 6, 1501727 (2016).

26. Hwang, J. Y., Myung, S. T. \& Sun, Y. K. Sodium-ion batteries: present and future. Chem. Soc. Rev. 46, 3529-3614 (2017).

27. Liang, J. et al. Could Li/Ni disorder be utilized positively? Combined experimental and computational investigation on pillar effect of $\mathrm{Ni}$ at $\mathrm{Li}$ sites on $\mathrm{LiCoO}_{2}$ at high voltages. Electrochim. Acta 146, 784-791 (2014).

28. Adamczyk, E. \& Pralong, V. $\mathrm{Na}_{2} \mathrm{Mn}_{3} \mathrm{O}_{7}$ : a suitable electrode material for $\mathrm{Na}$-ion batteries? Chem. Mater. 29, 4645-4648 (2017).

29. Zhao, X. et al Ab-initio investigations on bulk and monolayer $\mathrm{V}_{2} \mathrm{O}_{5}$ as cathode materials for Li-, Na-, K- and Mg-lon batteries. J. Mater. Chem. A 4, 16606-16611 (2016).

30. Zhang, X. et al. Tetragonal-structured anisotropic $2 \mathrm{D}$ metal nitride monolayers and their halides with versatile promises in energy storage and conversion. $J$. Mater. Chem. A 5, 2870-2875 (2017).

31. Liu, M. et al. Spinel compounds as multivalent battery cathodes: a systematic evaluation based on ab initio calculations. Energy Environ. Sci. 8, 964-974 (2015).

32. Li, G., Yue, X., Luo, G. \& Zhao, J. Electrode potential and activation energy of sodium transition-metal oxides as cathode materials for sodium batteries: a firstprinciples investigation. Comp. Mater. Sci. 106, 15-22 (2015).

33. Su, J., Pei, Y., Yang, Z. \& Wang, X. First-principles investigation on the structural, electronic properties and diffusion barriers of $\mathrm{Mg} / \mathrm{Al}$ doped $\mathrm{NaCoO}_{2}$ as the cathode material of rechargeable sodium batteries. RSC Adv. 5, 27229-27234 (2015).

34. $\mathrm{Fu}, \mathrm{S} ., \mathrm{Ni}, \mathrm{J} ., \mathrm{Xu}, \mathrm{Y}$., Zhang, Q. \& Li, L. Hydrogenation driven conductive $\mathrm{Na}_{2} \mathrm{Ti}_{3} \mathrm{O}_{7}$ nanoarrays as robust binder-free anodes for sodium-ion batteries. Nano. Lett. 16, 4544-4551 (2016).

35. Ni, J. et al. Superior sodium storage in $\mathrm{Na}_{2} \mathrm{Ti}_{3} \mathrm{O}_{7}$ nanotube arrays through surface engineering. Adv. Energy Mater. 6, 1502568 (2016).

36. Kresse, G. \& Furthmüller, J. Efficient iterative schemes for ab initio total-energy calculations using a plane-wave basis set. Phys. Rev. B 54, 11169-11186 (1996).

37. Blöchl, P. E. Projector augmented-wave method. Phys. Rev. B 50, 17953-17979 (1994).

38. Perdew, J. P., Burke, K. \& Ernzerhof, M. Generalized gradient approximation made simple. Phys. Rev. Lett. 77, 3865-3868 (1996).

39. Grimme, S., Antony, J., Ehrlich, S. \& Krieg, H. A consistent and accurate ab initio parametrization of density functional dispersion correction (DFT-D) for the 94 elements H-Pu. J. Chem. Phys. 132, 154104 (2010).

40. Grimme, S., Ehrlich, S. \& Goerigk, L. Effect of the damping function in dispersion corrected density functional theory. J. Comput. Chem. 32, 1456-1465 (2011).

41. Henkelman, G. \& Jónsson, H. Improved tangent estimate in the nudged elastic band method for finding minimum energy paths and saddle points. J. Chem. Phys. 113, 9978-9985 (2000).

42. Belsky, A., Hellenbrandt, M., Karen, V. L. \& Luksch, P. New developments in the Inorganic Crystal Structure Database (ICSD): accessibility in support of materials research and design. Acta Crytallogr. Sect. B 58, 364-369 (2002).

(c) (i) Open Access This article is licensed under a Creative Commons By Attribution 4.0 International License, which permits use, sharing, adaptation, distribution and reproduction in any medium or format, as long as you give appropriate credit to the original author(s) and the source, provide a link to the Creative Commons license, and indicate if changes were made. The images or other third party material in this article are included in the article's Creative Commons license, unless indicated otherwise in a credit line to the material. If material is not included in the article's Creative Commons license and your intended use is not permitted by statutory regulation or exceeds the permitted use, you will need to obtain permission directly from the copyright holder. To view a copy of this license, visit http://creativecommons. org/licenses/by/4.0/.

(c) The Author(s) 2018 